\title{
Comparison of quantitative measurements between two different intravascular ultrasound catheters and consoles: in vitro and in vivo studies
}

\author{
Hiroyuki Okura ${ }^{1,2}$ D $\cdot$ Makoto Watanabe ${ }^{1} \cdot$ Akihiro Miura $^{3} \cdot$ Muneo Kurokawa $^{3} \cdot$ Tomoya Ueda $^{1} \cdot$ Tsunenari Soeda $^{1}$. \\ Yoshihiko Saito ${ }^{1}$
}

Received: 8 October 2020 / Accepted: 19 January 2021 / Published online: 27 February 2021

(c) The Author(s) 2021

\begin{abstract}
Previous studies suggested possible discordant quantitative measurements between different IVUS catheters and/or systems. The purpose of this study was to assess compatibility of two different IVUS catheters and consoles for quantitative measurements of coronary arteries. (1). In vitro study: IVUS imaging was performed in a concentric cylindrical phantom with 6 sections of known, cross-sectional diameter ranging from 3.0 to $8.0 \mathrm{~mm}$. The lumen diameter (LD) and lumen cross-sectional area (LA) were measured and compared. To compare between 2 different IVUS consoles, IVUS images were obtained using a single IVUS catheter (catheter 1) connected to 2 different IVUS consoles (console 1 and 2). To compare between 2 different IVUS catheters, IVUS imaging was obtained using 2 different IVUS catheters (catheter 1 and 2) connected to a single IVUS console (console 2). (2). In vivo study: IVUS imaging was performed in 40 stented coronary arterial segments from 40 patients. The maximal stent diameter (Max SD), minimal stent diameter (minSD), and stent area (SA) were measured at both distal and proximal stent edges and compared between the two IVUS consoles (console 1 and 2) connected to a single IVUS catheter (catheter 1) $(n=20)$. IVUS imaging was also performed to compare between catheter 1 and 2 connected to IVUS console $2(n=20)$. Both in vitro and in vivo studies showed good correlation between the two IVUS consoles as well as two IVUS catheters. In conclusion, two IVUS catheters and consoles provide comparable IVUS measures both in vitro and in vivo.
\end{abstract}

Keywords Coronary artery disease $\cdot$ Intravascular ultrasound $\cdot$ Atherosclerosis $\cdot$ Stent

\section{Introduction}

Intravascular ultrasound (IVUS) is widely used to guide percutaneous coronary intervention (PCI) [1-5] and to assess efficacy of medication or PCI on coronary atherosclerotic plaque or stent in vivo [6-8]. Previously, there were discordance in quantitative measurements of the coronary arteries using different IVUS systems from different providers

Hiroyuki Okura

hokura@fides.dti.ne.jp

1 Department of Cardiovascular Medicine, Nara Medical University, Kashihara, Japan

2 Department of Cardiology, Gifu University Graduate School of Medicine, Gifu, Japan

3 Department of Medical Engineering, Nara Medical University, Kashihara, Japan mainly because of the differences in the speed of sound used in each IVUS system [9-11]. The aim of this study was to assess compatibility of two different IVUS catheters and consoles for quantitative measurements of coronary arteries.

\section{Methods}

\section{In vitro study}

IVUS imaging was performed in a concentric cylindrical phantom with 6 sections of known, cross-sectional diameter ranging from 3.0 to $8.0 \mathrm{~mm}$ as previously reported [12]. Images were obtained in a saline-filled tank maintained at $37{ }^{\circ} \mathrm{C}$. Commercially available IVUS catheters with a mechanical rotating $40 \mathrm{MHz}$ transducer (catheter 1) (ViewIT $^{\mathrm{TM}}$, Terumo Corporation, Tokyo, Japan) or $60 \mathrm{MHz}$ transducer (catheter 2) (AltaView ${ }^{\mathrm{TM}}$, Terumo Corporation, 
Tokyo, Japan) were used in this study. IVUS imaging was recorded using catheter 1 connected to either IVUS console 1 (VISIWAVETM, Terumo Corporation, Tokyo, Japan) or an IVUS console 2 (VISICUBETM, Ueda Japan Radio Co. Ltd, Nagano, Japan) to compare two IVUS consoles (Fig. 1, study 1). IVUS catheter was inserted sequentially into the distal edge of the tube and slowly pulled back automatically to the proximal edge. All procedures were performed by the same operator for both systems. In each tube, 5 arbitrary segments were selected for analysis. The lumen diameter (LD) and lumen cross-sectional area (LA) were measured and compared. Then, IVUS imaging was performed using IVUS catheter 2 (AltaView ${ }^{\mathrm{TM}}$ ) connected to console 2 (VISICUBE ${ }^{\mathrm{TM}}$ ) to compare two IVUS catheters (Fig. 1, study 2).
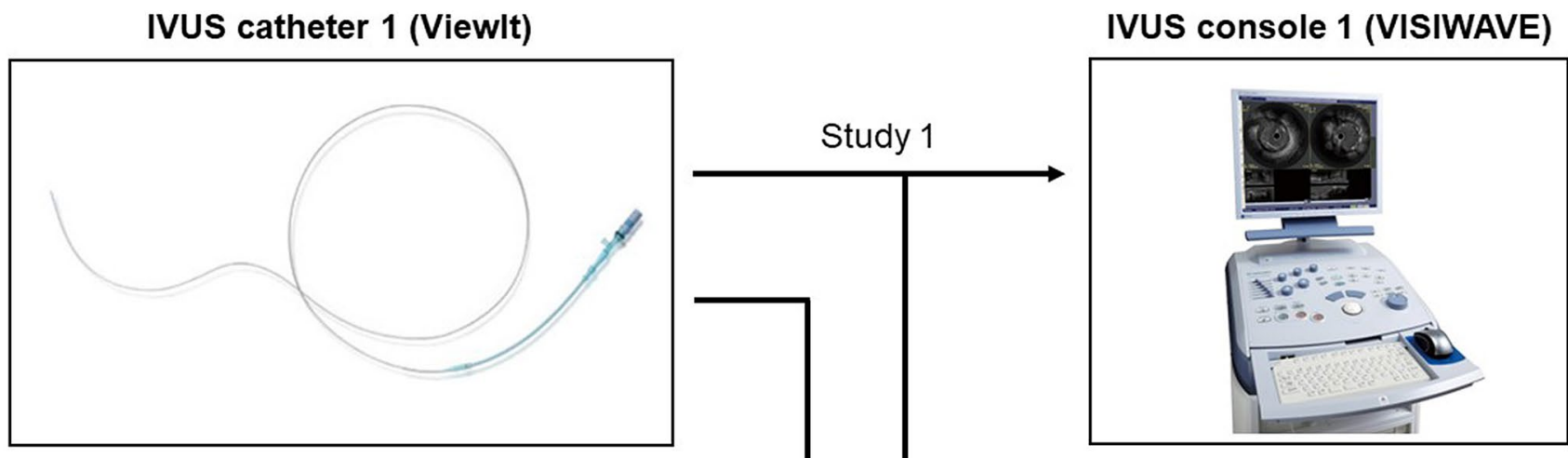

IVUS catheter 2 (AltaView)
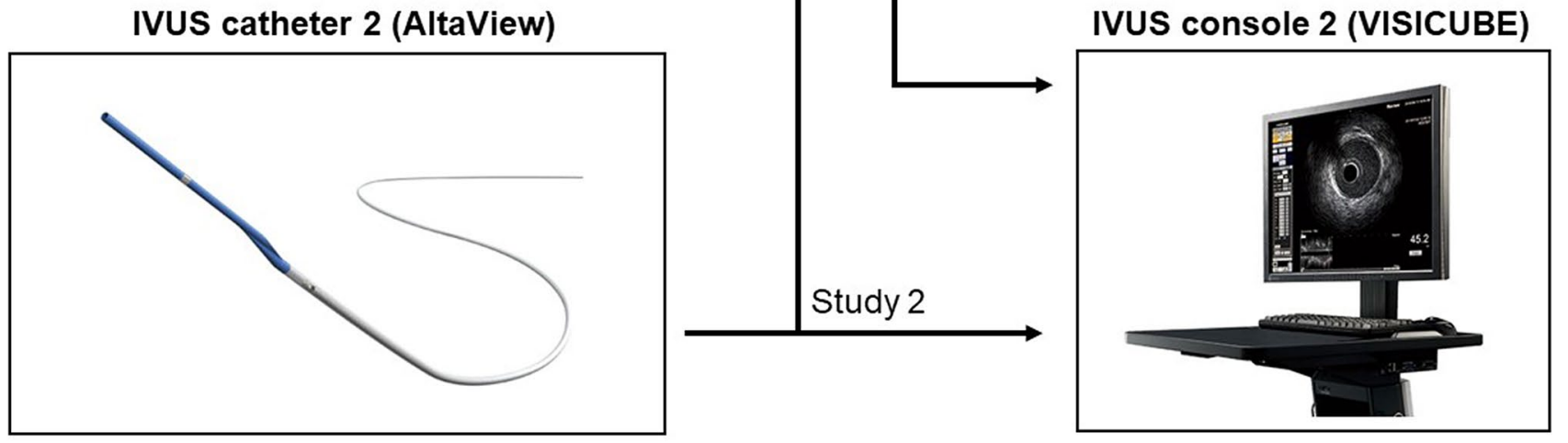

Fig. 1 IVUS catheters and consoles used in this study. Study 1 compared 2 consoles and study 2 compared 2 catheters
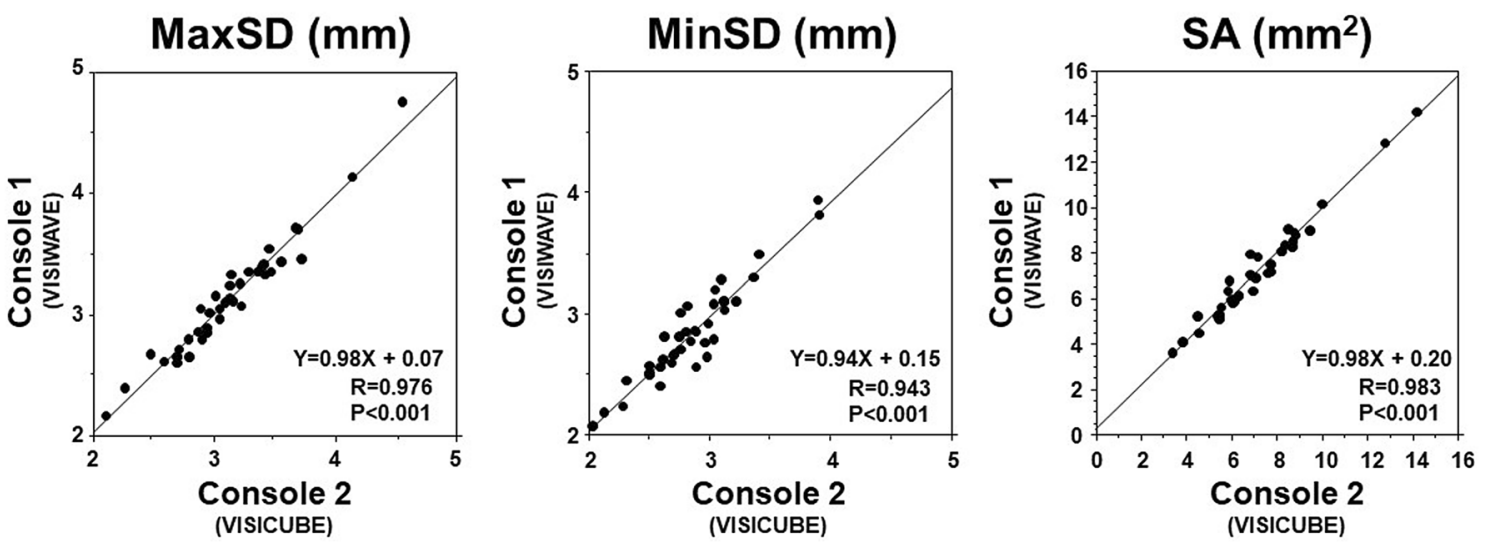

Fig. 2 Comparison between 2 different IVUS consoles (in vivo). MaxSD, MinSD, and SA showed good correlations between IVUS console 1 and 2 


\section{In vivo study}

Forty patients with native coronary artery disease who underwent stent implantation were enrolled in the in vivo study. The first 20 patients underwent IVUS imaging after PCI by IVUS catheter 1 (ViewIT ${ }^{\mathrm{TM}}$ ) connected to either IVUS console 1 (VISIWAVETM) or IVUS console 2 (VISI-

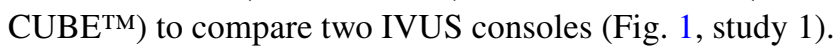
The next 20 patients underwent IVUS imaging after PCI by IVUS catheter 1 (ViewIT ${ }^{\mathrm{TM}}$ ) followed by IVUS catheter 2 (AltaView $^{\mathrm{TM}}$ ) connected to console 2 (VISIWAVE ${ }^{\mathrm{TM}}$ ) to compare two IVUS catheters (Fig. 1, study 2). Images with a significantly reduced quality were excluded. This study was in compliance with the Declaration of Helsinki with regard to investigations in humans, and the study protocol was approved by the Ethics Committee of Nara Medical University Hospital (Number 1278 and 1754). Written informed consent was obtained from all patients before cardiac catheterization and IVUS imaging.

\section{IVUS imaging and analysis}

IVUS pullback was performed using an automated pullback device at a rate of $0.5 \mathrm{~mm} / \mathrm{s}$ or $9.0 \mathrm{~mm} / \mathrm{s}$ (IVUS catheter 2 only). The IVUS images were continuously recorded on each IVUS console and then transferred to CD-ROM for offline analysis. The IVUS images recorded on CD-ROM were analyzed using a commercially available planimetry system (VISIATLAS ${ }^{\mathrm{TM}}$, Terumo Corporation, Tokyo, Japan) [13]. In the in vivo study, coronary stented segments were selected, and images of the proximal edges and distal edges were selected for cross-sectional measurement. Maximal stent diameter (MaxSD), minimal stent diameter (MinSD), and stent area (SA) were measured at both distal and proximal stent edges (both study 1 and 2). Using an automated pullback device at a rate of $0.5 \mathrm{~mm} / \mathrm{s}$ (both catheter 1 and 2) or $9.0 \mathrm{~mm} / \mathrm{s}$ (catheter 2 only), total stent length (TSL) was measured and compared between the 2 systems (study 2 ).

\section{Statistical analysis}

Statistical analysis was performed with StatView version 5.0 (SAS Institute, Cary, NC, USA). Sample size was determined based on the previous similar studies [10,12]. Continuous variables are reported as mean $\pm \mathrm{SD}$. The comparisons between two different IVUS catheters or consoles were performed using linear regression analysis. A $P$ value of $<0.05$ was considered to be significant.
Table 1 Comparison between IVUS measurements and true value (in vitro study)

Table 2 Comparison between 2 different IVUS catheters (in vitro study)

Table 3 Comparison between 2 different IVUS consoles (in vitro study)

\begin{tabular}{lllllr}
\hline Catheter & Console & Measurements & Correlation formula & $R$ & $P$ value \\
\hline ViewIT & VISIWAVE & LD & $Y=1.00 X+0.05$ & 0.9999 & $<0.001$ \\
& & LA & $Y=0.99 X-0.27$ & 0.9999 & $<0.001$ \\
\multirow{2}{*}{ ViewIT } & VISICUBE & LD & $Y=1.00 X-0.02$ & 0.9999 & $<0.001$ \\
& & LA & $Y=1.01 X-0.18$ & 0.9999 & $<0.001$ \\
AltaView & VISICUBE & LD & $Y=1.01 X-0.11$ & 0.9998 & $<0.001$ \\
& & LA & $Y=1.00 X-0.57$ & 0.9998 & $<0.001$ \\
\hline
\end{tabular}

$L A$ lumen area, $L D$ lumen diameter

\begin{tabular}{lllllll}
\hline Catheter 1 & Catheter 2 & Console & Measurements & Correlation formula & $R$ & $P$ value \\
\hline \multirow{2}{*}{ ViewIT } & AltaView & \multirow{2}{*}{ VISICUBE } & LD & $Y=1.00 X+0.05$ & 0.9999 & $<0.001$ \\
& & & LA & $Y=0.99 X-0.27$ & 0.9999 & $<0.001$ \\
\hline
\end{tabular}

$L A$ lumen area, $L D$ lumen diameter

\begin{tabular}{lllllll}
\hline Catheter & Console 1 & Console 2 & Measurements & Correlation formula & $R$ & $P$ value \\
\hline \multirow{2}{*}{ ViewIT } & \multirow{2}{*}{ VISICUBE } & \multirow{2}{*}{ VISIWAVE } & LD & $Y=1.00 X-0.03$ & 0.9998 & $<0.001$ \\
& & & LA & $Y=1.01 X-0.21$ & 0.9997 & $<0.001$ \\
\hline
\end{tabular}

$L A$ lumen area, $L D$ lumen diameter 


\section{Results}

\section{In vitro study}

Both LD and LA obtained by the two IVUS systems correlated well with the actual size of the tube (Table 1, Fig. S1). Comparison between two different IVUS catheters is shown in Table 2. Two different catheters provide similar quantitative measures. Comparison between two different IVUS consoles is shown in Table 3. IVUS measurements obtained by a single IVUS catheter connected to two different IVUS consoles provide similar results.

\section{In vivo study}

Figure 2 shows the correlation between the values from catheter 1 (ViewIT ${ }^{\mathrm{TM}}$ ) with console 1(VISIWAVE ${ }^{\mathrm{TM}}$ ) vs. catheter1 (ViewIT ${ }^{\mathrm{TM}}$ ) with console 2 (VISICUBE ${ }^{\mathrm{TM}}$ ). MaxSD $(R=0.976, P<0.001), \operatorname{minSD}(R=0.943, P<0.001)$, and SA $(R=0.983, P<0.001)$ obtained by the two IVUS consoles have a good correlation. Figure 3 shows the correlation between the values from catheter 1 (ViewIT ${ }^{\mathrm{TM}}$ ) with console 2 (VISICUBE ${ }^{\mathrm{TM}}$ ) and catheter 2 (AltaView $^{\mathrm{TM}}$ ) with console 2 (VISICUBETM). MaxSD $(R=0.982, P<0.001)$, $\operatorname{minSD}(R=0.976, P<0.001)$, and SA $(R=0.996, P<0.001)$ obtained by the two IVUS catheters connected to the same console (console 2) have a good correlation.

IVUS-derived TSL measured by catheter 1 at a rate of $0.5 \mathrm{~mm} / \mathrm{s}$ and catheter 2 at a rate of $0.5 \mathrm{~mm} / \mathrm{s}$ showed a good correlation $(R=0.994, P<0.001)$ (Fig. 4). Similarly, TSL by catheter 1 at a rate of $0.5 \mathrm{~mm} / \mathrm{s}$ and catheter 2 at
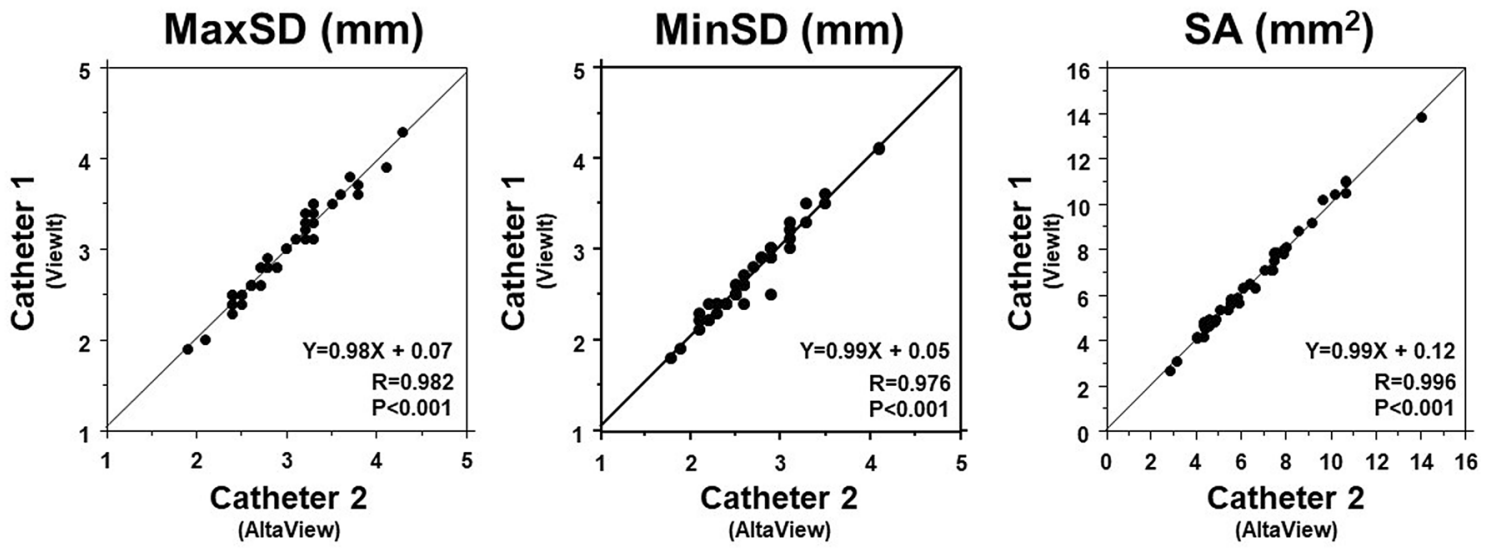

Fig. 3 Comparison between 2 different IVUS catheters (in vivo). MaxSD, MinSD, and SA showed good correlations between IVUS catheter 1 and 2

Fig. 4 Comparison of IVUSderived total stent length (TSL) between 2 catheters. TSL obtained by IVUS catheter 1 at a rate of $0.5 \mathrm{~mm} / \mathrm{s}$ correlated well with TSL obtained by catheter 2 at a rate of $0.5 \mathrm{~m} / \mathrm{s}$ (left panel). TSL obtained by IVUS catheter 1 correlated well with TSL by IVUS catheter 2 at a rate of $9.0 \mathrm{~mm} / \mathrm{s}$
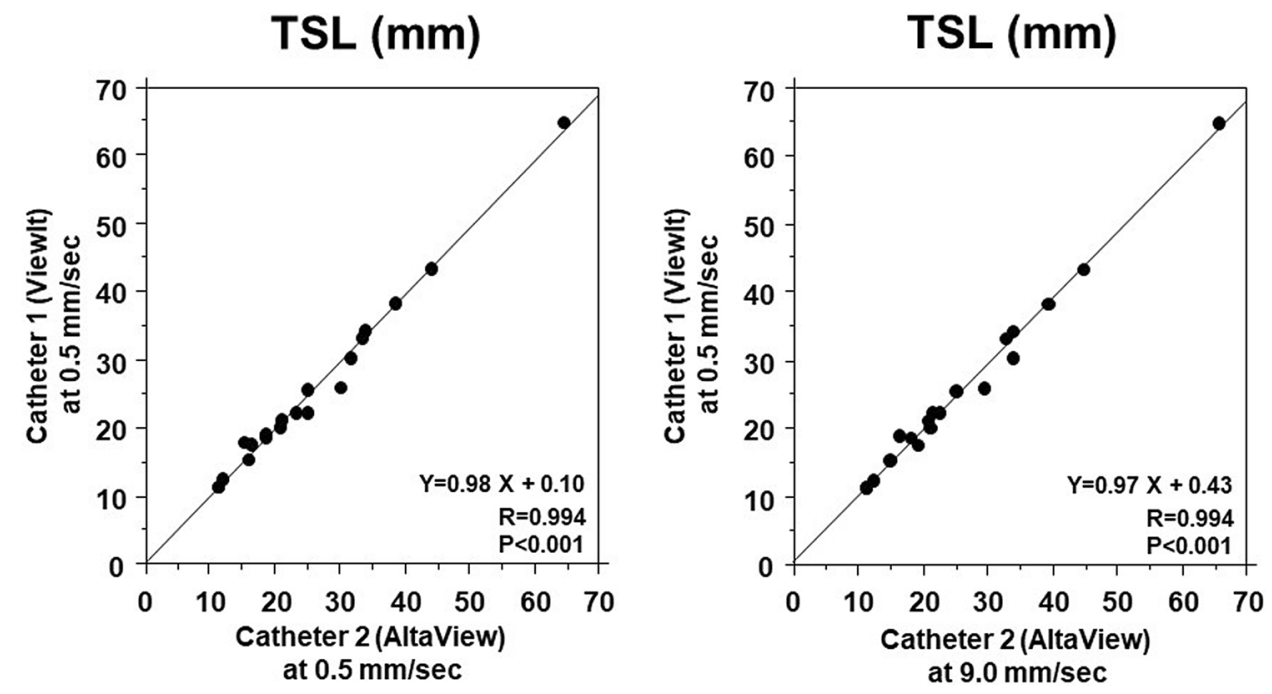
a rate of $9.0 \mathrm{~mm} / \mathrm{s}$ showed a good correlation $(R=0.994$, $P<0.001$ ) (Fig. 4). In addition, TSL by catheter 1 at a rate of $0.5 \mathrm{~mm} / \mathrm{s}$ and catheter 1 at a rate of $9.0 \mathrm{~mm} / \mathrm{s}$ showed a good correlation $(Y=1.02 \mathrm{X}-0.46, R=0.996, P<0.001$, figure not shown).

\section{Discussion}

Our study demonstrated that a new IVUS catheter as well as IVUS console provide accurate quantitative measurements. In vitro study demonstrated that a new IVUS catheter and IVUS console provide quantitative measures comparable to a previously validated IVUS catheter and IVUS console. In addition, two IVUS consoles with different pullback device provided comparable length measurements. These results suggest that currently available IVUS system can be used during PCI procedures as well as clinical research similar to the previous version of the IVUS catheter and console.

Currently, IVUS has been routinely used during PCI[3-5]. IVUS provides quantitative measures to select device size and to optimize PCI results $[1,4]$. We do believe that IVUSderived measurements are accurate and reproducible. However, early studies demonstrated discrepancies in quantitative results between different IVUS systems [9-11]. An in vitro study comparing between $40 \mathrm{MHz}$ mechanical IVUS (Atlantis SR/Ultracross, Boston Scientific) and $20 \mathrm{MHz}$ phased array IVUS (InVision/Avanar, Volcano, Rancho Cordova, CA, USA) demonstrated up to $18 \%$ differences in quantitative results between the 2 systems [9]. Similarly, an ex vivo human coronary artery study showed that plaque area and plaque burden were significantly larger in mechanical IVUS (Atlantis SR) than phased array IVUS (InVision)[11]. Even between similar IVUS systems (mechanical IVUS) $(40 \mathrm{MHz}$ Atlantis versus $35 \mathrm{MHz}$ Intrafocus, Terumo), in vitro as well as in vivo study demonstrated that lumen diameter, lumen area, plaque plus media area, and external elastic membrane area by one IVUS (Intrafocus) were significantly smaller than those obtained by another IVUS (Atlantis)[10]. Difference in the speed of ultrasound used in each IVUS system $(1530 \mathrm{~m} / \mathrm{s}$ in Intrafocus and $1562 \mathrm{~m} / \mathrm{s}$ in Atlantis) and the frequency of the transducer $(35 \mathrm{MHz}$ in Intrafocus and $40 \mathrm{MHz}$ in Atlantis) are suspected as possible causes of the differences in quantitative measurements [12]. Because the second-generation IVUS catheters and systems used similar frequencies and the same a speed of sound, quantitative measurements between different IVUS systems should be comparable. Indeed, a previous study comparing two different IVUS systems from different companies demonstrated comparable quantitative measures [12]. Yamada et al. compared two different mechanical rotating IVUS catheters (Atlantis Pro2 ${ }^{\mathrm{TM}}$, Boston Scientific Corporation, Natick, MA, USA and ViewIT TM, Terumo Corporation,
Tokyo, Japan) and showed that these 2 different commercially available IVUS systems were accurate and comparable. Recently, a brand new IVUS catheter and console with higher frequency and higher pullback speed (AltaView and VISICUBE) has become clinically available. Although theoretically the new IVUS catheter and console would provide comparable quantitative measures, validation study is needed. As expected, our data confirmed that the new IVUS catheter and IVUS console provided accurate and comparable results compared with older IVUS catheter and IVUS console. Because ViewIT and VISIWAVE have not been completely replaced by the AltaView and VISWAVE, it is important to demonstrate the compatibility of either IVUS catheter or IVUS console. Indeed, ViewIT is still on the market and is used with either VISIWAVE or VISICUBE. In this regard, our results have strong clinical implications with respect of the reliability of the quantitative IVUS measures during IVUS-guided PCI. Furthermore, IVUS is also used to assess serial changes in coronary vessel, plaque as well as stent to evaluate efficacy of drug and stent itself $[6-8,14$, 15]. Therefore, our results have implication, showing that quantitative data obtained from different IVUS catheters and consoles could be used.

Previous studies demonstrated that length measurements by IVUS using motorized pullback of the transducer and imaging core through a stationary imaging sheath provide accurate length and thus have been clinically utilized $[16,17]$. These studies used constant pullback at a rate of $0.5 \mathrm{~mm} / \mathrm{s}$ because of limited frame rate (30 frames $/ \mathrm{s}$ ). Because of the variable and higher frame rates (30-90 frames/s) used in the newer IVUS console (VISICUBE), IVUS imaging can be performed at variable and higher pullback speed $(0.5-9.0 \mathrm{~mm} / \mathrm{s})$. Therefore, we also compared stent TSL between standard pullback speed $(0.5 \mathrm{~mm} / \mathrm{s})$ and higher pullback speed $(9.0 \mathrm{~mm} / \mathrm{s})$, and demonstrated that stent length measurements can be reliably performed using higher pull back speed at a rate of $9.0 \mathrm{~mm} / \mathrm{s}$. These results also have an important clinical implication during PCI. With use of fast pullback before PCI, we could avoid risk of ischemia during IVUS pullback and shorten procedure time. Although faster pullback speed may be advantageous for accurate measurements, because it is less affected by cardiac motion, our results do not indicate that one is better than the other. Larger sample size with variable vessels would be necessary to answer this question.

Our current study has several limitations. First, we compared between 2 catheters and consoles from a single company. Therefore, it is not conclusive that the newer IVUS catheter (AltaView) and a console (VISICUBE) provide similar quantitative data compared with another IVUS systems from different companies. Second, we only imaged and measured stented segment to minimize the risk of complications by performing multiple IVUS pullback imaging before 
treatment. Therefore, it is uncertain if pre-intervention IVUS-derived quantitative measures are comparable [2]. In particular, quantitative measurements, especially length measurements based on the IVUS pullback recording, may be affected by the tortuosity of the vessel. Results of our present study assessing the stented segments only may not be applicable to the tortuous vessel. Third, we performed only single IVUS imaging pullback recording with each IVUS catheter by a single operator; therefore, the impact of the IVUS imaging method such as order of catheter insertion or differences in insertion/recording technique by each operator could not be evaluated. However, it is unlikely that quantitative results are affected by them. Finally, qualitative assessment of the coronary plaque was not compared between the 2 catheters and 2 consoles, because this was not the purpose of this study. Comparison of visually assessed plaque types or IVUS-derived tissue characterization (IB-IVUS) would be required to answer this question [18-21].

In conclusion, a new version of IVUS catheter and console provide accurate and comparable quantitative measures as compared with a previous IVUS catheter and console. Therefore, these two catheters and consoles are exchangeable and could be comparably used during IVUS-guided PCI and IVUS-based clinical investigations.

Supplementary Information The online version contains supplementary material available at https://doi.org/10.1007/s12928-021-00759-6.

Acknowledgements This study was, in part, supported by Terumo Corporation. Otherwise, all authors have no conflict of interest or financial disclosure regarding this manuscript.

Open Access This article is licensed under a Creative Commons Attribution 4.0 International License, which permits use, sharing, adaptation, distribution and reproduction in any medium or format, as long as you give appropriate credit to the original author(s) and the source, provide a link to the Creative Commons licence, and indicate if changes were made. The images or other third party material in this article are included in the article's Creative Commons licence, unless indicated otherwise in a credit line to the material. If material is not included in the article's Creative Commons licence and your intended use is not permitted by statutory regulation or exceeds the permitted use, you will need to obtain permission directly from the copyright holder. To view a copy of this licence, visit http://creativecommons.org/licenses/by/4.0/.

\section{References}

1. Mintz GS, Nissen SE, Anderson WD, Bailey SR, Erbel R, Fitzgerald PJ, et al. American College of Cardiology Clinical Expert Consensus Document on Standards for Acquisition, Measurement and Reporting of Intravascular Ultrasound Studies (IVUS). A report of the American College of Cardiology Task Force on Clinical Expert Consensus Documents. J Am Coll Cardiol. 2001;37(5):1478-92.

2. Okura H, Morino Y, Oshima A, Hayase M, Ward MR, Popma JJ, et al. Preintervention arterial remodeling affects clinical outcome following stenting: an intravascular ultrasound study. J Am Coll Cardiol. 2001;37(4):1031-5.

3. Okura H, Saito Y, Soeda T, Nakao K, Ozaki Y, Kimura K, et al. Frequency and prognostic impact of intravascular imaging-guided urgent percutaneous coronary intervention in patients with acute myocardial infarction: results from J-MINUET. Heart Vessels. 2019;34(4):564-71.

4. Saito Y, Kobayashi Y, Fujii K, Sonoda S, Tsujita K, Hibi K, et al. Clinical expert consensus document on standards for measurements and assessment of intravascular ultrasound from the Japanese Association of Cardiovascular Intervention and Therapeutics. Cardiovasc Interv Ther. 2020;35(1):1-12

5. Sonoda S, Hibi K, Okura H, Fujii K, Honda Y, Kobayashi Y. Current clinical use of intravascular ultrasound imaging to guide percutaneous coronary interventions. Cardiovasc Interv Ther. 2020;35(1):30-6.

6. Nissen SE, Tuzcu EM, Libby P, Thompson PD, Ghali M, Garza $D$, et al. Effect of antihypertensive agents on cardiovascular events in patients with coronary disease and normal blood pressure: the CAMELOT study: a randomized controlled trial. JAMA. 2004;292(18):2217-25.

7. Takagi T, Okura H, Kobayashi Y, Kataoka T, Taguchi H, Toda I, et al. A prospective, multicenter, randomized trial to assess efficacy of pioglitazone on in-stent neointimal suppression in type 2 diabetes: POPPS (Prevention of In-Stent Neointimal Proliferation by Pioglitazone Study). JACC Cardiovasc Interv. 2009;2(6):524-31.

8. Nicholls SJ, Puri R, Anderson T, Ballantyne CM, Cho L, Kastelein JJ, et al. Effect of evolocumab on progression of coronary disease in statin-treated patients: the GLAGOV randomized clinical trial. JAMA. 2016;316(22):2373-84.

9. Schoenhagen P, Sapp SK, Tuzcu EM, Magyar WA, Popovich J, Boumitri M, et al. Variability of area measurements obtained with different intravascular ultrasound catheter systems: Impact on clinical trials and a method for accurate calibration. J Am Soc Echocardiogr. 2003;16(3):277-84.

10. Li Y, Honye J, Saito S, Takayama T, Yokoyama S, Saruya T, et al. Variability in quantitative measurement of the same segment with two different intravascular ultrasound systems: in vivo and in vitro studies. Catheter Cardiovasc Interv. 2004;62(2):175-80.

11. Hartmann M, von Birgelen C, Mintz GS, Deppermann N, Dirsch $\mathrm{O}$, Stoel MG, et al. Dedicated calibration formulas permit correction of differences between measurements by different IVUS devices as demonstrated in atherosclerotic human coronary arteries in vitro. Int J Cardiovasc Imaging. 2006;22(5):605-13.

12. Yamada R, Okura H, Kume T, Hayashida A, Neishi Y, Kawamoto $\mathrm{T}$, et al. Comparison of quantitative measurements between two different intravascular ultrasound systems: in vitro and in vivo studies. J Cardiol. 2013;61(3):201-5.

13. Nakayama N, Hibi K, Endo M, Miyazawa A, Suzuki H, Maejima $\mathrm{N}$, et al. Validity and reliability of new intravascular ultrasound analysis software for morphological measurement of coronary artery disease. Circ J. 2013;77(2):424-31.

14. Ako J, Hibi K, Kozuma K, Miyauchi K, Morino Y, Shinke T, et al. Effect of alirocumab on coronary atheroma volume in Japanese patients with acute coronary syndromes and hypercholesterolemia not adequately controlled with statins: ODYSSEY J-IVUS rationale and design. J Cardiol. 2018;71(6):583-9.

15. Ako J, Hibi K, Tsujita K, Hiro T, Morino Y, Kozuma K, et al. Effect of alirocumab on coronary atheroma volume in japanese patients with acute coronary syndrome-the ODYSSEY J-IVUS trial. Circ J. 2019;83(10):2025-33.

16. Fuessl RT, Mintz GS, Pichard AD, Kent KM, Satler LF, Popma JJ, et al. In vivo validation of intravascular ultrasound length measurements using a motorized transducer pullback system. Am J Cardiol. 1996;77(12):1115-8. 
17. Tanaka K, Carlier SG, Mintz GS, Sano K, Liu X, Fujii K, et al. The accuracy of length measurements using different intravascular ultrasound motorized transducer pullback systems. Int J Cardiovasc Imaging. 2007;23(6):733-8.

18. Yamada R, Okura H, Kume T, Neishi Y, Kawamoto T, Watanabe $\mathrm{N}$, et al. Histological characteristics of plaque with ultrasonic attenuation: a comparison between intravascular ultrasound and histology. J Cardiol. 2007;50(4):223-8.

19. Miyamoto Y, Okura H, Kume T, Kawamoto T, Neishi Y, Hayashida A, et al. Plaque characteristics of thin-cap fibroatheroma evaluated by OCT and IVUS. JACC Cardiovasc Imaging. 2011;4(6):638-46.

20. Yamada R, Okura H, Kume T, Neishi Y, Kawamoto T, Miyamoto $\mathrm{Y}$, et al. A comparison between $40 \mathrm{MHz}$ intravascular ultrasound
iMap imaging system and integrated backscatter intravascular ultrasound. J Cardiol. 2013;61(2):149-54.

21. Kobayashi Y, Okura H, Kume T, Miyamoto Y, Yamada R, Kobayashi $\mathrm{Y}$, et al. Direct relationship of local C-reactive protein production and lipid pool characterized by integrated backscatter intravascular ultrasound: a preliminary observation. Coron Artery Dis. 2015;26(5):425-31.

Publisher's Note Springer Nature remains neutral with regard to jurisdictional claims in published maps and institutional affiliations. 